\title{
Propuesta de Modelo para el Proceso de Enseñanza- Aprendizaje Colaborativo de la Observación en Diseño, utilizando la Pizarra Digital Interactiva (PDI)
}

\author{
Juan C. Briede( ${ }^{(1)}$, Isabel M. Leal(1), Marcela L. Mora ${ }^{(2)}$ y Claudia S. Pleguezuelos ${ }^{(2)}$ \\ (1) Universidad del Bío-Bío, Departamento de Arte y Tecnologías del Diseño, Casilla 5-C, Concepción- \\ Chile (e-mail: jbriede@ubiobio.cl; ileal@ubiobio.cl). \\ (2) Universidad del Bío-Bío, Área de Desarrollo Pedagógico y Tecnológico, Coordinación Unidad de \\ Gestión Curricular y Monitoreo, Casilla 447, Chillán-Chile (e-mail: mamora@ubiobio.cl; \\ cpleguez@ubiobio.cl).
}

Recibido Ago. 8, 2014; Aceptado Oct. 23, 2014; Versión final recibida Dic. 22, 2014

\section{Resumen}

Se presenta una propuesta basada en el uso de la pizarra digital interactiva como apoyo al desarrollo de la observación y su dimensión creativa, dentro del proceso de enseñanza/aprendizaje del diseño en cursos universitarios. En la formación del diseñador industrial en la Universidad del Bío-Bío se enfatiza la observación como método para asistir a las diversas fases del proyecto de diseño, comunicándola a través del discurso lingüístico (palabra) y del visual (croquis). Debido a la complejidad multimodal de ambos lenguajes, los docentes requieren tiempo de conversación colaborativa para interpretar, corregir e intervenir con sugerencias, dificultándose el registro y consignación formal de la síntesis y conclusiones que emergen del diálogo. La pizarra digital interactiva (pdi) como plataforma de proyección pública, pantalla interactiva y registro a través del almacenamiento digital, aporta a la sociabilización e inmediatez dialéctica, favoreciendo también la retroalimentación de los conceptos, croquis y proceso creativo.

\section{Proposed Model for Collaborative Teaching-Learning of Observation in Design Using the Interactive Digital Whiteboard (IDW)}

\begin{abstract}
The present article introduces a new application of the interactive digital whiteboard, which supports the development of creative observation during the process of teaching and learning in the field of Design. The training of Industrial Designers at the University of Bío-Bío (among other universities) emphasizes observation as a key method used throughout project design, specifically applied to linguistic (word) and visual (sketch) discourse. Given the multimodal complexity of both languages, teachers require the time to engage in collaborative discussion with students, interpreting, correcting and providing suggestions. It is often difficult, however, to register and formally appropriate the summary and conclusions that emerge from this dialogue. The interactive digital whiteboard (idw) acts as a platform for public viewing, as well as an interactive screen that is capable of digitally registering information. It supports socialization as well dialectical immediacy, and favors concept feedback, sketches and the overall creative process.
\end{abstract}

Keywords: teaching-learning, digital interactive whiteboard, industrial design, creativity 


\section{INTRODUCCIÓN}

El proceso de enseñanza/aprendizaje del diseño, en concordancia con la naturaleza de la disciplina, ha tenido como eje rector el desarrollo proyectual. Desde el punto de vista pedagógico, la carrera de diseño industrial, utiliza el enfoque que está más cerca del aprendizaje basado en proyecto (ABP), que si bien es similar al aprendizaje basado en problemas (Smith et al., 2005), comparten la misma abreviación, trabajan con desafíos del mundo real y consideran a un facilitador (Thomas,2000).

El proyecto de diseño busca integrar aspectos, tanto teórico-conceptuales como socio-técnicos, dentro de un período académico, para obtener un producto de diseño como resultado. La observación en este contexto es transversal a todas las fases del proyecto, actuando en su inicio para la detección de problemáticas y oportunidades en contextos reales, luego, como sustento de argumentación y búsqueda teórica, y posteriormente, para la materialización del objeto-solución. La enseñanza/aprendizaje con este método no prescriptivo, y que se realiza como acto personal, está basado en lo que el observador, en este caso el estudiante, genere. También el método permite que los efectos tecnológicos de globalización e interconexión se incorporen a las alternativas de comunicación y sociabilización, condicionando y afectando los hábitos tradicionales. La adaptación e integración de dichas tecnologías en la didáctica es una vía para apoyar los procesos de aprendizaje efectivos en las nuevas generaciones, cuyas prácticas y hábitos están en la categoría de "nativos digitales" (Prensky, 2001), y permite una mejor aproximación a la enseñanza del diseño basada en la observación, que tradicionalmente se encuentra asociada a un hacer análogo (lápiz y papel).

El Taller, como asignatura, donde se desarrolla el proyecto, tradicionalmente utiliza el aula como espacio de reunión presencial y sincrónica para abordar este proceso en forma colectiva. Se ha detectado que en las sesiones colectivas, donde los alumnos presentan las láminas con observaciones, existe mucho conocimiento relevante que surge del diálogo, y que se evidencia en tono de sugerencias, observaciones, conclusiones, y otros actos de habla, pero son pocos los alumnos que realizan registro de estas conversaciones y los que lo hacen, en muchos casos sólo quedan a modo de notas personales.Por lo demás, la generación de notas y correcciones sobre la propia lámina se percibe como una acción invasiva. Esta situación problemática ofrece la oportunidad de utilizar la pantalla digital con dos propósitos :primero, que el alumno proyecte su lámina y que los docentes puedan hacer retroalimentación sobre la misma, y segundo, que dicho registro quede como respaldo, tanto para el estudiante como para el grupo de profesores. Es así que los contrastes entre los lenguajes oral-visual, análogo-digital, teórico-práctico, metodológico-discursivo, se convierten en un reto que a continuación se pretende afrontar.

\section{LA OBSERVACIÓN COMO MÉTODO DE ENSEÑANZA/APRENDIZAJE}

El acto de observación en la Escuela de Diseño Industrial de la Universidad del Bío-Bío se enseña y aplica desde primer año, y se ha elegido como principio y método de búsqueda, tanto intelectual como creativa, en la firme creencia que fomentar la experiencia observadora (Cruz, 2010) desarrolla en los estudiantes la reflexión y la capacidad crítica ante lo cotidiano. Además, aplicada como método, la observación es lo suficientemente flexible como para complementarse con cualquier otra estrategia metodológica (Briedey Mora,2013) o incluso técnica, que se considere necesaria para dar cumplimiento a las metas que el diseñador se proponga. Su potencial radica en develar lo no evidente entre los actos humanos y su entorno, un buen observador atiende los detalles que finalmente pueden conformar el verdadero aspecto relevante de un acto, acción, objeto o relación, determinando de esta manera lo que más tarde será lo que conforme el valor de un diseño. Es entonces, un acto de búsqueda del conocimiento que se encarna y manifiesta en la obra de diseño, y que no es posible lograr sin involucrarse, sin la relación personal, racional y emotiva del sujeto que observa. Todo esto convierte a la observación en un acto transversal, marcado por tres dimensiones: a) filosófica (pensamiento), b) sintaxis (metodología y su orden), y c) praxis (acción y actitud).

\section{a) Filosofía de la observación}

En su dimensión filosófica, la observación se aproxima a la fenomenología, porque no distingue límites entre el sujeto que observa y lo observado, como tampoco entre este último y todo el sistema que lo rodea. Para Husserl, fundador del pensamiento fenomenológico, observar no es sólo un acto sensorial, como tampoco únicamente racional o intelectual, es una vivencia trascendente porque es conocimiento del sujeto que conoce (Husserl, 1986), podemos decir entonces que puede dar origen al pensamiento totalizante y creativo. Para Varela es la manera en que el objeto surge como fruto de nuestra actividad, por lo que tanto el objeto como la persona están co-emergiendo, co-surgiendo (Varela, 2000). Luis Oyarzún denomina esta instancia como contemplación, es decir, como el momento de plenitud y de trascendencia que nos distingue como seres humanos y nos hace ser libres y creadores, la contemplación no es sólo apertura cognoscitiva a un objeto, que tiende a una máxima amplitud; no es sólo concentración y absorción en él. Es también 
actividad, expresión constructora (Oyarzún, 1981); por ello el arte, y toda actividad creadora, forma parte de la expresión libre y trascendente de este acto contemplativo.

El principio esencial que sustenta a la observación es la suspensión del juicio, lo que motiva el nacimiento de una acción desde un sentir que no juzga y que es netamente humanista, porque son actos de personas, tanto en su actuar como en sus relaciones con el entorno, con otros y consigo mismo, por lo tanto, los conceptos que surgen son aquellos que nos unen como seres humanos, y por ello pueden ser comprendidos. La selección del mundo observado implica el compromiso con el acto y la identificación del mismo observador, quien, en este instante de suspensión de juicio, revela estas relaciones para profundizar en ellas. El método creativo ya no es sólo un protocolo mecánico, ni una técnica, sino que surge como la reflexión del observar, que se respalda en el diálogo y se comunica por medio del lenguaje, sea éste verbal o no.

Desde esta perspectiva, la palabra es el conector de la idea o concepto rescatado en la observación, que en el acto dialógico se convierte en una forma expresiva, un sentido, pero también es la expresión del Ser que la nombra, Heidegger (1995) en el texto "La palabra, la significación de las palabras", declara que la palabra origina el lenguaje, y la palabra unifica al Ser del hombre y su destino, es la voz del Ser que devela su verdad, tanto en lo dicho como en lo no dicho, puesto que se rescata del devenir humano. La palabra nos acerca como modo expresivo a la poética, porque se apropia del mundo, ya que poetizar es predecir, como un Decir inicial que acontece, pero que no es dependiente de lo hecho en el presente y lo efectivo en él (Op cit, 1995), entonces, saber decir lo observado poéticamente permite al mismo tiempo aprehender la observación vivenciada como un acto creador, lo que posteriormente se expresará en un lenguaje creativo del diseño; es una idea proyectada basada en las relaciones del ser humano en su plenitud actuante y existencial, pero que surge y se concreta en una nueva expresión separada de la norma y que convoca a un nuevo sentido del ser y del actuar, por ello es creación pura. En la práctica, en una exposición en cualquier Taller de Diseño, donde se les solicita a los alumnos exponer frente a una lámina sus observaciones, implica que cada estudiante debe coordinar la palabra dicha (oral), la palabra escrita (presentada como concepto escrito) y la "palabra" dibujada o en algunos casos, esquematizada, pero no sólo eso, sino que además, implica el discurso de los énfasis realizados con la voz y los gestos. Todos estos lenguajes unificados requieren interpretación de parte de los docentes en lo dicho, pero también en lo no dicho, y como tal complejidad, también se realiza en la retroalimentación, no resulta extraño suponer que gran parte de los signos emitidos durante este diálogo espontáneo se omita. La Pizarra digital permite entonces rescatar y retener buena parte de lo más relevante del acto discursivo, jerarquizando y concentrando a manera de huella, el recuerdo del proceso y por ende, del potencial acto creativo producto del encuentro.

\section{b) La sintaxis de la observación}

Como ya se ha dicho, la observación requiere primeramente ser un acto presencial, es por lo tanto, netamente perceptivo, pero posee un proceso de profundización e inmersión en el acto que involucra un tipo de selección basada en las relaciones, es decir, abrirse a nuevas formas de entendimiento sobre lo que se observa, para ello requiere evidenciar por medio de registros que expongan y construyan sus propias huellas, uno de los elementos más relevantes y próximos que asisten al proceso cognitivo del observador en el diseño es el croquis, útil al momento de plasmar y exteriorizar, así como también para compartir y comunicar a una audiencia lo que más tarde constituirá el respaldo de lo detectado como valor, y para ello se debe ejercitar la forma y la palabra.

Esta suerte de simbiosis o integración de lenguajes permite en el acto de la observación, ir construyendo la visión del mundo, de lo percibido por parte del observador. Un proceso dialéctico (Goldschmidt, 1991) que supone el acto mismo de abocetar o croquear y de la palabra que explica y define. El croquis como imagen permite representar cualidades formales, atributos espaciales y establecer relaciones en un contexto espacial. Por ello la observación, permite definir las cualidades y singularidades de lo percibido y el croquis tiene el propósito de contextualizarlo, acotarlo y escalarlo a una realidad física. En dicho formato se registran a mano alzada las ideas utilizando el lenguaje escrito y croquis que buscan contextualizar en un entorno físico dichas relaciones. Las figuras 1,2 y 3 presentan las observaciones realizadas por alumnos de la carrera, durante el proceso de análisis del contexto.

Se puede ver en estos ejemplos la relevancia de la visualización y la palabra que conecta la idea o concepto, rescatado desde la observación, con el acto comunicativo y dialógico, afiata y destaca, conformado de manera más clara no sólo lo observado, sino que al observador que lo costruye, puesto que es él quien ha decidido rescatar con la palabra los elementos de un devenir humano específico, cerrando el ciclo creativo del pensamiento generado en la observación, que en combinación con la imagen, es aprehender la realidad, su apropiación del mundo que posteriormente se expresará en el lenguaje del 
diseño. La palabra y la imagen que dentro del proceso del Taller, son los dos lenguajes que permitirán avanzar en la didáctica del diseño.

Los entes participantes fluyen por un espacio acotado, donde el cliente momentáneo invade el área del consumidor pasivo.

De preferencia es requerido el sector más apartado de la puerta, que es donde se encuentra el mayor flujo de personas.

La cercanía de las mesas conlleva la invasión de la privacidad de cada grupo.

Las personas ocupan el espacio desde la periferia hasta el centro, convocando una circulación en espiral dentro del establecimiento.

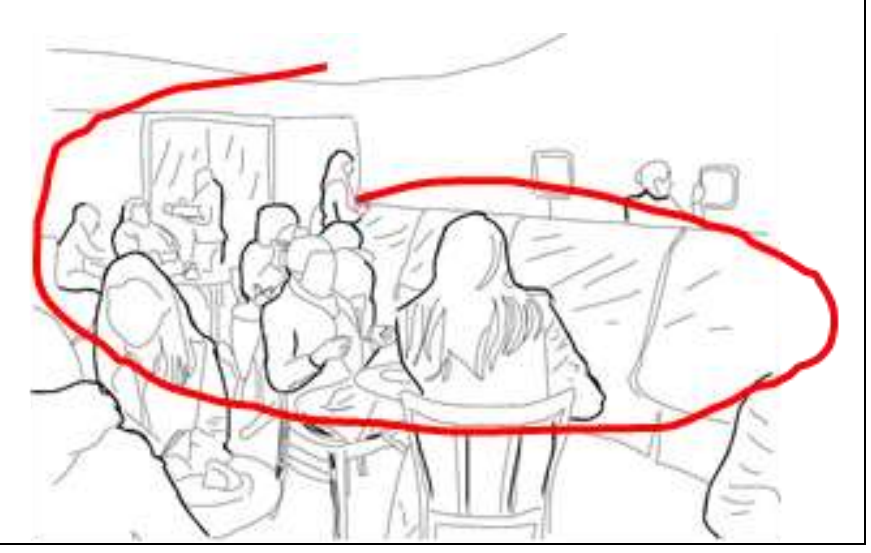

Fig. 1. Ejemplo de lámina con observación. Observaciones realizadas en una cafetería para una empresa de Concepción. Elaboración Margarita Hidalgo y Evelyn Bastías, Taller IV, 2011

Discontinuidad productiva determinada por la extracción manual generando tiempos muertos.

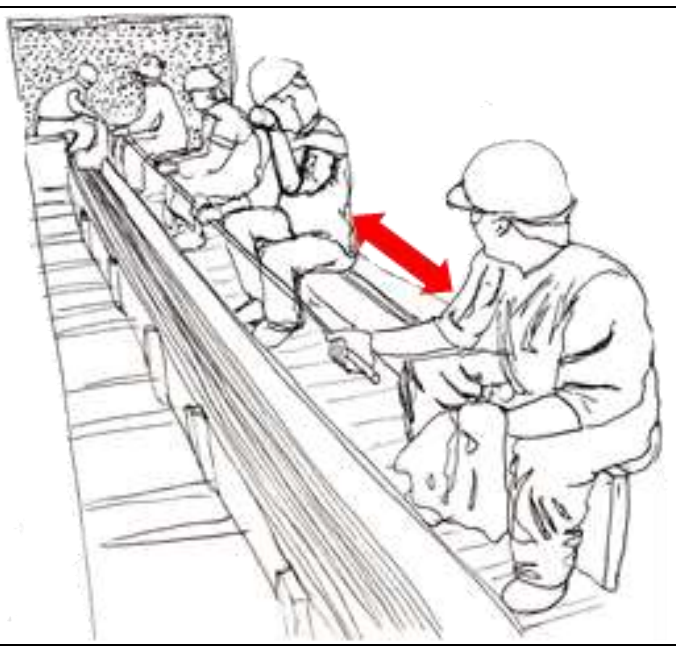

Fig. 2. Observación realizada en una empresa donde se trabaja en extracciones de tubos de transferencia térmica para una industrial de Concepción. Elaboración Felipe Fica, Taller IV, 2011

La complejidad de la extracción de tubos determina interdependencia de los operarios en el proceso.

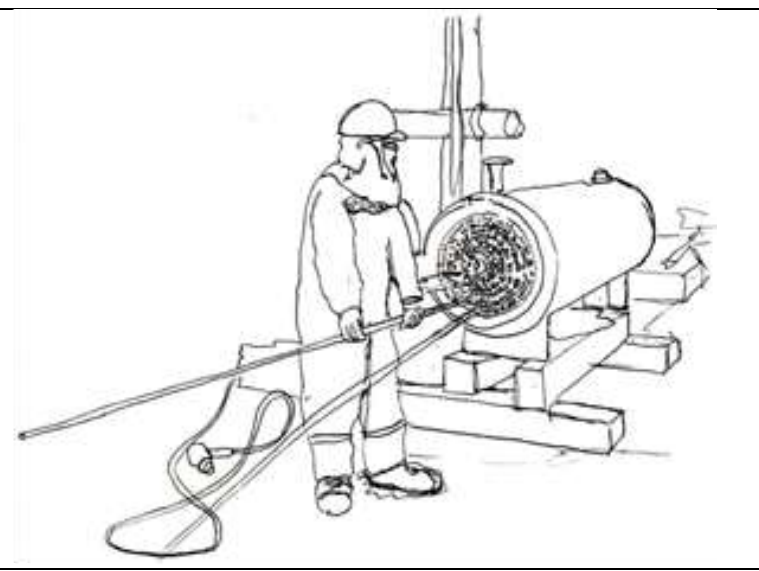

Fig. 3.Observación realizada en una empresa donde se trabaja en extracciones de tubos de transferencia térmica para una industrial de Concepción. Elaboración Felipe Fica, Taller IV, 2011

\section{c) La praxis de la observación}

El espacio de enseñanza/aprendizaje que otorga el aula permite congregar y trabajar colectivamente ciertos procesos. Existe una serie de interfaces o modos en los cuales el docente se interrelaciona con los 
estudiantes. Según Mabardi (2012), esto se produce a través de distintos tipos de careos entre profesores y estudiantes y diversos encuentros. En este modelo se proponen correcciones intermedias colectivas que permiten generar un epicentro de discusión. A partir del diálogo se va construyendo el conocimiento y la propia observación. Para hablar de su praxis es necesario profundizar en el espacio Taller.

\section{EL TALLER, ESPACIO COLECTIVO}

En este proceso se distingue el grado de implicación de los presentes. La corrección de las observaciones se genera en un espacio colectivo del aula por lo que los alumnos implícitamente van a participar, opinando y aportando con un punto de vista complementario o antagónico. En esta propuesta se busca desarrollar un proceso colaborativo en el que los individuos involucrados se comprometan o tengan una actitud colaborativa frente al estudiante que está presentando su trabajo y busca obtener una retroalimentación que se traducirá en una guía, un refuerzo o corrección del proceso presentado. En ese sentido los alumnos tienen que adquirir un rol particular pero parcial dentro del contexto colectivo, el que permita aclarar y fundar un nuevo conocimiento colectivo.

Es un proceso dialógico. El diálogo en el proceso creativo de un Taller es fundamental; para ello es preciso aclarar que desde una perspectiva comunicacional puede entenderse como consenso, donde su producto es una creación que convocó a los participantes para una acción interactiva, generada desde y a partir del diálogo, proceso considerado no sólo como una capacidad verbal, sino que también visual (por medio de croquis, bosquejos, láminas y modelaciones virtuales, registros audiovisuales, entre otros medios), volumétrica (a través de la visualización de modelos de intención, relaciones entre partes y piezas, materialidades), asimismo lo vital de considerar al usuario por medio del análisis gestual, y la mímesis de la acción que, en muchos casos, se produce entre objeto y sujeto. Este tipo de relaciones puede ser ejemplificada en las figuras 1, 2,3 donde los diversos usuarios interactúan con entidades objetuales con el propósito de ejecutar una acción.

Al ser consensual, el respeto resulta un valor trascendente, y si bien en el acto de observar ya se debía aplicar la suspensión del juicio, en el acto dialógico es una condicionante, puesto que la obra surge desde la construcción individual pero se desarrolla en el colectivo, y es en este punto donde cada sujeto aporta desde sus competencias y áreas de conocimiento, lo que lo convierte en una actividad fácilmente adaptada a lo interdisciplinario. Para ello, la comunicación y los estados de confianza que se practiquen antes son fundamentales, porque en las instancias creadoras los participantes y las ideas o potenciales acciones son consideradas y puestas en análisis, independiente de su fuente, porque todas son igualmente válidas, todo con el fin de experimentar y mejorar un producto. Por esto mismo, en el evento comunicativo es necesario dejar de lado las estrategias discursivo-persuasivas u otras forma de retórica que impliquen actos de poder, convencimiento o dominancia, puesto que los actos deben depender sólo de la libertad creadora y de una relación de igualdad y plena confianza entre los participantes.

\section{MODELO PROPUESTO}

El presente modelo procura integrar el uso de la pizarra digital para apoyar el proceso de sociabilización y el de registro de las retroalimentaciones sobre el trabajo del alumno. Y se estructura en las etapas que se presentan en la Tabla 1.

En dicho modelo se busca potenciar el uso de la Pizarra Digital Interactiva (PDI) en la Etapa Final del Ciclo, que es la de Sociabilización. Este insumo busca amplificar los hallazgos del alumno dentro de un espacio colectivo, facilitando la visualización (posibilita amplificar los detalles del material) y la percepción de los alumnos del taller. Ya que el formato de entrega está concebido para ser percibido y leído a una distancia cercana, lo que propicia un espacio más íntimo de interacción. El énfasis de este modelo está en la posibilidad de generar una retroalimentación sobre el material de la misma presentación y además de obtener un registro y respaldo de dichas aportaciones para ser enviados tanto al alumno en cuestión como poder tener un respaldo dentro de los archivos del Taller para seguimiento y monitoreo de dicho aprendizaje. Este proceso se representa en el diagrama de flujo que se muestra en la figura 4.

En los últimos años la revolución tecnológica ha influido ampliamente en la manera de vivir, lo que también ha impactado en la manera de enseñar y aprender (Baelo y Cantón, 2009). Existen herramientas y recursos tecnológicos que posibilitan estrategias didácticas apoyadas en estos medios para facilitar la construcción de conocimientos (Esteve y Gisbert, 2011), sin embargo, como afirma Maznah, (2006, p. 83) el uso exitoso de la PDI no trata de la tecnología, sino de integrar la tecnología en el plan de estudios para crear experiencias de aprendizaje personalizado para cada alumno y transformar el aula en un entorno de aprendizaje colaborativo (citado en Northcote et al.,2010). 
Tabla1: Modelo Proceso Enseñanza/Aprendizaje de la Observación

\begin{tabular}{|c|c|c|c|}
\hline Nombre etapa & & Modalidad de trabajo & Insumo \\
\hline Presentación & $\begin{array}{l}\text { - Estructura Observación (discurso verbal + } \\
\text { discurso visual) } \\
\text { - Relaciones o interacciones entre entidades } \\
\text { (Usuarios-gente, objetos, espacios) } \\
\text { - Criterios y parámetros observados. (Obs. aplicada } \\
\text { al diseño) } \\
\text { - Ejemplos }\end{array}$ & Colectivo & Pizarra Digital \\
\hline $\begin{array}{l}\text { Encargo } \\
\text { Observación }\end{array}$ & $\begin{array}{l}\text { - Contexto, acotación } \\
\text { - Número o cantidad de láminas } \\
\text { - Formato de presentación de lámina } \\
\text { - Discurso escrito } \\
\text { - Croquis (técnica) }\end{array}$ & Colectivo & $\begin{array}{l}\text { Pizarra } \\
\text { Convencional } \\
\text { PPT }\end{array}$ \\
\hline Nombre etapa & & Modalidad de trabajo & Insumo \\
\hline Trabajo autónomo & $\begin{array}{l}\text { - Trabajo de Campo } \\
\text { - Visitar contexto de estudio } \\
\text { - Construcción observaciones en contexto } \\
\text { - Registro, notas }\end{array}$ & Individual & Croquera \\
\hline Sociabilización & $\begin{array}{l}\text { - Presentación de hallazgos } \\
\text { - Diálogo reflexivo argumentativo } \\
\text { - Recomendaciones } \\
\text { - Conclusiones }\end{array}$ & Colectivo & Pizarra Digital \\
\hline
\end{tabular}

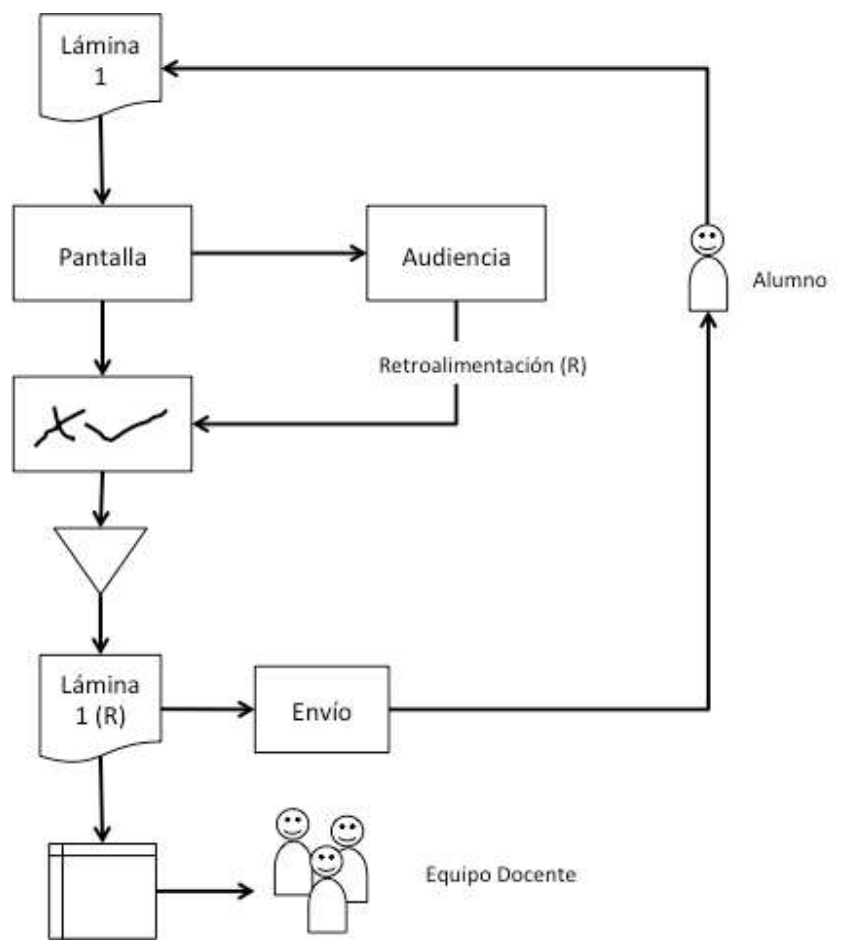

Fig. 4. Diagrama del proceso con el uso de la pizarra digital interactiva.

El presente modelo busca integrar el uso de la pizarra digital interactiva para apoyar el proceso de sociabilización de las presentaciones colectivas, de la puesta en común de las diversas etapas del proyecto de diseño y el registro de las retroalimentaciones sobre el trabajo del alumno en colaboración con sus pares y profesor. Dorado (2011) afirma que: "la incorporación de esta sencilla y asequible tecnología, en sus diversas modalidades, así como la facilidad de su uso, permite a los agentes participantes la doble perspectiva de la acción y la reflexión, tanto del análisis y evaluación del proceso de generación, como de 
los resultados obtenidos, favoreciendo así las dinámicas colaborativas, metacognitivas y de generación del conocimiento. El conocimiento basado en los procesos de corrección y de registro que permite la PDI, considera no sólo la interacción de los profesores con los alumnos (o entre los alumnos), sino que una autoevaluación que permite la posibilidad de infinitas modificaciones sustentadas en tres lenguajes: la palabra, el gesto y lo visual (en los bosquejos y croquis), lo que facilita enormemente el proceso creativo e iterativo que supone.

Gallego y Gatica (2010), describen la pizarra digital interactiva como un sistema tecnológico generalmente integrado por un ordenador, un video proyector y un dispositivo de control de puntero, que permite proyectar *en una superficie interactiva* contenidos digitales en formato idóneo para visualización en grupo. Se puede interactuar directamente sobre la superficie de proyección. Los autores destacan como una ventaja para los alumnos, que lo que aparece en la pantalla, textos, dibujos, imágenes, esquemas, puede imprimirse, reduciéndose la necesidad de tomar apuntes.

Se han realizado diversas revisiones bibliográficas sobre el uso de la PDI, que dan cuenta de sus potencialidades como recurso tecnológico para la enseñanza y el aprendizaje. Entre los recogidos por Gandol et al. (2012), se destaca lo que sigue:

La PDI es un medio efectivo para revisar sesiones anteriores al mismo tiempo que ayuda en el manejo de la clase y su comportamiento gracias a su flexibilidad, versatilidad y la posibilidad de realizar aprendizaje colaborativo (Bell, et al., 2007; Mohon, 2008; O'Hanlon, 2007; Somyurek et al., 2009). Al mismo tiempo, permite el conocimiento compartido y que todos los alumnos vean las contribuciones de los demás (Mohon, 2008; Vincent, 2007)

Coscollola, (2011), por su parte, revisa varias investigaciones en el contexto español que apoyan el empleo de la PDI, donde se afirma que facilitan la posibilidad de usar más recursos, potencian la motivación y participación del alumnado, y favorecen la comprensión de los temas. Similares aseveraciones ya se habían encontrado en el contexto anglosajón a través del informe de investigación de Becta (2003), donde se señalaba como beneficios clave de la PDI, el aumento de la motivación y la generación de mayor oportunidad para que los alumnos participen y colaboren. Entre las características metodológicas atribuidas a la PDI se menciona, además, que permite unas dinámicas de aprendizaje centradas en el alumno y en las que el profesor adopta un rol de facilitador del aprendizaje (O'Hanlon, 2007; Wall e t al., 2005; Wood \& Ashfield, 2008; citado en Gandol, F. et al. 2012) y la posibilidad de corregir errores en los materiales (Beauchamp \& Parkinson, 2005). Además se ha encontrado evidencia del uso de la PDI como una herramienta para fomentar y apoyar el diálogo en el aula (Mercer et al.,2010; Hennessy, 2011) y del valor de esta herramienta para orquestar múltiples modos semióticos y apoyar la negociación de significados de los diferentes aspectos de una tarea (Fernández-Cárdenas, 2013)

\section{Metodología de la investigación:}

El proyecto de intervención educativa con el uso de la pizarra digital se orientó con la pregunta de investigación: ¿Qué recursos didácticos-evaluativos y tecnológicos permiten orientar en forma más efectiva el proceso de diseño relevando la observación como competencia de los diseñadores industriales? Se desarrolló una metodología cualitativa, con técnicas de grupo focal como inicio, para levantar información acerca de las prácticas que los docentes desarrollan en el taller de diseño y sus principales dificultades a la hora de retroalimentar las propuestas de proyectos de diseño de los estudiantes. Posteriormente se realizó una exhaustiva búsqueda bibliográfica del uso de la pizarra digital en contexto de educación universitaria y se adscribió al enfoque de evaluación auténtica. Se realizó una prueba donde se aplicó el modelo propuesto en la figura 4. en una sesión de corrección de la asignatura Taller. Esta sesión fue registrada en video con objeto de tener el material visual y auditivo para realizar los posteriores análisis del proceso dialógico entre estudiantes y docentes usando la pizarra digital, para lo cual se aplicó la técnica de análisis crítico del discurso, indicando las acciones, gestos, marcadores y paralenguaje del Acto de Habla y la participación de la Pizarra digital en dicho acto, para finalmente, emitir los comentarios sobre sus relaciones.

\section{A continuación, un ejemplo de la actividad:}

El contexto del siguiente fragmento es la actividad que ocurre en la asignatura de Taller de tercer año de la carrera de Diseño Industrial en la Universidad del Bío-Bío y corresponde a un momento dentro del proceso de corrección dialógica centrada en una lámina expuesta (presentada y proyectada usando la pizarra digital), por un grupo de alumnos. Participan 4 docentes y 4 alumnos y durante la actividad se encuentran presentes otros compañeros de curso, que trabajan en silencio y en ciertos momentos están atentos a lo que se expone, pero no intervienen. Temática y académicamente, el ejemplo se refiere a la etapa de observación (lo que indica que aún falta para que realicen propuestas de forma), los alumnos analizan el 
proceso productivo de las artesanas que trabajan en el área textil, se focalizan especialmente en las acciones y gestos que realizan al momento de sacar y guardar los retazos de tela.

La columna izquierda que se titula "Interacción del estudiante/docente" es una transcripción de la actividad, la columna titulada "paralenguaje" son comentarios e inferencias de los gestos, proxémica y kinésica realizada, la columna titulada "pizarra digital" son comentarios e inferencias sobre el papel que en ese momento cumple la mediación de la plataforma y por último, la columna "comentarios y relaciones", son comentarios del análisis en relación con lo dicho y observado sobre la pizarra digital en el acto discursivo.

\section{RESULTADOS}

Ejemplo de retroalimentación con la Pizarra Digital en proceso de croquis de la observación.

\begin{tabular}{|l|l|}
\hline \multicolumn{2}{|l|}{ Desgrabación observación de video para retroalimentación de croquis utilizando pizarra digital } \\
\hline Contextualización & Taller Diseño centrado en el usuario \\
\hline Curso & $\begin{array}{l}\text { Diseñar un producto basado en necesidades y requerimientos de usuarios reales } \\
\text { (artesano) aplicando metodologías centradas en el usuario. }\end{array}$ \\
\hline Resultado de Aprendizaje & Equipo de trabajo $N^{\circ} 2$ \\
\hline Grupo de Trabajo & Diseño croquis para evidenciar problemática \\
\hline Actividad de Aprendizaje & Presentación de croquis y solución a la problemática \\
\hline Actividad de Evaluación &
\end{tabular}

Desgrabación y transcripción del Acto de Habla (5 minutos 17 segundos de la actividad)

\begin{tabular}{|c|c|c|c|}
\hline Interacción del estudiante/docente & Paralenguaje & Pizarra Digital & $\begin{array}{l}\text { Comentarios } \quad y \\
\text { relaciones }\end{array}$ \\
\hline $\begin{array}{l}\text { Estudiante: Hace referencia al contexto de } \\
\text { su proyecto, señala la problemática. (por } \\
\text { motivos de que no interviene el docente } \\
\text { con los registro de la PDI, este apartado se } \\
\text { excluye } \\
\text { Docente 1: Hacia arriba, dibuja y deja el } \\
\text { registro en el croquis, mediante recurso } \\
\text { Pizarra Digital. }\end{array}$ & $\begin{array}{l}\text { El Docente, señala (indica) donde se } \\
\text { evidencia el problema, esa observación } \\
\text { queda registrada en la pizarra digital por } \\
\text { parte del estudiante } 1 .\end{array}$ & $\begin{array}{l}\text { La frase del profesor } \\
\text { apela e induce al } \\
\text { alumnos a "rayar" o } \\
\text { "marcar" la lámina, con } \\
\text { la finalidad de resaltar el } \\
\text { espacio (que supone ser } \\
\text { uno de los aspectos de } \\
\text { jerarquía mayor dentro } \\
\text { del discurso de } \\
\text { observación). }\end{array}$ & $\begin{array}{l}\text { Comienza a activarse la } \\
\text { participación de la PDi, } \\
\text { primero como índice (no } \\
\text { registrado, sólo señalado). }\end{array}$ \\
\hline $\begin{array}{l}\text { Estudiante 1: (Indica cuál sería el espacio } \\
\text { y señala) este sería el espacio. }\end{array}$ & $\begin{array}{l}\text { El alumno señala y raya la lámina } \\
\text { dejando registro mediante PDi }\end{array}$ & $\begin{array}{l}\text { Dicha observación } \\
\text { permitirá al estudiante } \\
\text { reorientar su proceso de } \\
\text { diseño, reelaborando } \\
\text { parte del croquis para } \\
\text { expresar mejor la idea } \\
\text { de la problemática y la } \\
\text { solución a ésta. }\end{array}$ & $\begin{array}{l}\text { El acto de rayar la lámina } \\
\text { refuerza la jerarquía de ese } \\
\text { espacio dentro del discurso, } \\
\text { por lo tanto, la importancia, } \\
\text { que supondrá en el futuro. }\end{array}$ \\
\hline $\begin{array}{l}\text { Estudiante 3: ...60x55 sería el espacio } \\
\text { ese... a ... noo, pero que eso está en } \\
\text { normado en la maqueta así } \\
\text { pero...(estudiante } 1 \text { señala la maqueta } \\
\text { ubicada en la mesa)... }\end{array}$ & $\begin{array}{l}\text { El alumno comenta acercarse a la lámina, } \\
\text { mirando al alumno que expone y a los } \\
\text { docentes, el estudiante } 1 \text { le "ofrece" el } \\
\text { lápiz. (La observación está relacionada } \\
\text { con una maqueta 3D entregada y que se } \\
\text { encuentra sobre una mesa, para } \\
\text { complementar el discurso y que indica } \\
\text { medidas y orden) }\end{array}$ & $\begin{array}{l}\text { La Pizarra no es activa } \\
\text { en ese momento porque } \\
\text { se centra la atención } \\
\text { sobre la maqueta, sin } \\
\text { embargo el Estudiante } 1 \\
\text { se mantiene en su } \\
\text { posición de expositor } \\
\text { líder, aunque señala la } \\
\text { maqueta) }\end{array}$ & $\begin{array}{l}\text { La pizarra aún se encuentra } \\
\text { con poca alteración, sólo } \\
\text { mantiene el registro de la } \\
\text { marca del espacio señalado. }\end{array}$ \\
\hline $\begin{array}{l}\text { Estudiante 2: Pero en la maqueta de } \\
\text { contexto está normado el espacio y eso } \\
\text { para que podamos trabajar y eso }\end{array}$ & $\begin{array}{l}\text { El alumno comenta sin acercarse a la } \\
\text { lámina. }\end{array}$ & $\begin{array}{l}\text { Se centra la atención } \\
\text { sobre la maqueta, }\end{array}$ & $\begin{array}{l}\text { La pizarra aún se mantiene } \\
\text { inactiva }\end{array}$ \\
\hline
\end{tabular}




\begin{tabular}{|c|c|c|c|}
\hline Interacción del estudiante/docente & Paralenguaje & Pizarra Digital & Comentarios y relaciones \\
\hline $\begin{array}{l}\text { Docente 1: De alguna manera esto ... } \\
\text { (lo indica en la proyección de la } \\
\text { lámina) (se dirige a la sala) Shhh... } \\
\text { silencio por favor...esta sería la línea } \\
\text { límite } \\
\text { ¿no? Sería una pared }\end{array}$ & $\begin{array}{l}\text { El docente se aproxima más a la proyec-ción, } \\
\text { confronta al alumno, solicitándole el lápiz con } \\
\text { un gesto de la mano, raya la lámina dejando } \\
\text { registro mediante PDi. La ilocución al expo- } \\
\text { nerse en tono de pregun-ta, en una compren- } \\
\text { sión efectiva de la "inocencia" frente a la duda } \\
\text { y minimiza el acto "violento" que implica rayar } \\
\text { el traba-jo. También confronta al resto del cur- } \\
\text { so para que mantenga silencio durante la co- } \\
\text { rrección, una vez realizado el rayado le entre- } \\
\text { ga el lápiz al estudiante } 1 \text { y se sienta a } \\
\text { escuchar. }\end{array}$ & $\begin{array}{l}\text { Se centra la atención } \\
\text { sobre la lámina proyec- } \\
\text { tada y el espacio indica- } \\
\text { do por el profesor,en } \\
\text { ese momento, las } \\
\text { alteraciones realizadas } \\
\text { son para una mejor } \\
\text { comprensión de imagen } \\
\text { 2D con el fin de facilitar } \\
\text { "imaginarlo" en 3D. Se } \\
\text { refuerza la } \\
\text { representación total. }\end{array}$ & $\begin{array}{l}\text { La pizarra se activa, con el } \\
\text { rayado y permite "adecuar" } \\
\text { la idea representada desde } \\
\text { lo bidimensional a lo } \\
\text { tridimensional. } \\
\text { Se corrige la representación } \\
\text { para facilitar el lenguaje y la } \\
\text { comunicación. }\end{array}$ \\
\hline $\begin{array}{l}\text { Docente 2.: Pared, y luego una } \\
\text { ventana ¿cierto?... (asienten } \\
\text { estudiante } 1 \text { y docente 1) a la altura de } \\
\text { la mesa (risas y el estudiante } 1 \\
\text { refuerza con un gesto de aprobación) }\end{array}$ & $\begin{array}{l}\text { El docente } 2 \text { no se aproxima a la pizarra ni } \\
\text { raya la lámina, pero le hace comentarios } \\
\text { desde su espacio (a un metro y medio aprox. } \\
\text { de la proyección) para aclarar los elementos } \\
\text { que se configuran en la lámina. } \\
\text { El alumno indica la lámina dejando registro } \\
\text { mediante PDi }\end{array}$ & $\begin{array}{l}\text { El estudiante } 1 \text {, toma la } \\
\text { iniciativa y mientras el } \\
\text { docente } 2 \text { realiza sus } \\
\text { comentarios, comienza } \\
\text { a rayar la lámina para } \\
\text { "corregir" los espacios } \\
\text { indicados en el croquis. }\end{array}$ & $\begin{array}{l}\text { Es interesante que el acto } \\
\text { de rayar la lámina por parte } \\
\text { del estudiante es inducido } \\
\text { por el docente, quien } \\
\text { mientras más interviene y } \\
\text { raya, más posiciona al } \\
\text { alumno sobre su acto de } \\
\text { representación y su } \\
\text { autoridad sobre el croquis. }\end{array}$ \\
\hline $\begin{array}{l}\text { Docente 3: La ventana que está } \\
\text { indicada... }\end{array}$ & $\begin{array}{l}\text { El docente } 3 \text {, tampoco se aproxima a la } \\
\text { pizarra ni raya la lámina, pero le hace } \\
\text { comentarios señalando y relacionando lámina } \\
\text { con maqueta. }\end{array}$ & $\begin{array}{l}\text { El docente relaciona lo } \\
\text { proyectado bidimensio- } \\
\text { nalmente con lo repre- } \\
\text { sentado a escala, } \\
\text { tridimensionalmente. }\end{array}$ & $\begin{array}{l}\text { La pizarra se mantiene } \\
\text { inactiva, sólo participa como } \\
\text { referencia }\end{array}$ \\
\hline $\begin{array}{l}\text { Docente 1: Es importante eso (le } \\
\text { solicita el lápiz al alumno y marca la } \\
\text { imagen mientras habla) en el fondo } \\
\text { que aparezca en el espacio (raya) .. y } \\
\text { después .. ¿está por acá? Ahí, está la } \\
\text { ventana (Dibuja la idea señalada). El } \\
\text { croquis no sólo debe mostrar las cosas } \\
\text { evidentes como una foto, sino que tú } \\
\text { puedes usar recursos como transpa- } \\
\text { rencias, vistas fantasmas, cortes para } \\
\text { dar a entender cuál es la situación... } \\
\text { donde ocurre todo el problema. (le } \\
\text { entrega el lápiz al estudiante 1) }\end{array}$ & $\begin{array}{l}\text { El docente se aproxima a la proyección, y le } \\
\text { solicita el lápiz con un gesto de la mano, raya } \\
\text { la lámina dejando registro mediante PDi. La } \\
\text { ilocución se realiza en tono correctivo, pero el } \\
\text { acto de rayado es para corroborar la } \\
\text { interpretación, solicitando retroalimentación } \\
\text { de parte del estudiante, escribe y proyecta } \\
\text { para mostrar otras maneras de graficar, una } \\
\text { vez realizado el rayado le entrega el lápiz al } \\
\text { estudiante } 1 \text { y se sienta a escuchar. }\end{array}$ & $\begin{array}{l}\text { La lámina es rayada } \\
\text { para fortalecer el len- } \\
\text { guaje tridimensional y } \\
\text { los espacios no declara- } \\
\text { dos (lo no dicho y que } \\
\text { es necesario decir y } \\
\text { reforzar para un mejor } \\
\text { entendimiento), facilitan- } \\
\text { do la comprensión de } \\
\text { espacios y movimientos } \\
\text { que se deben imaginar } \\
\text { los interpretes del } \\
\text { discurso. }\end{array}$ & $\begin{array}{l}\text { La Pizarra y la lámina } \\
\text { entonces funciona no sólo } \\
\text { para comunicar la idea } \\
\text { inicial, sino que ayuda a } \\
\text { "corregir" (como acto de } \\
\text { metalenguaje) los aspectos } \\
\text { técnicos de la misma } \\
\text { representación, quedando } \\
\text { en registro los textos y las } \\
\text { alternativas. }\end{array}$ \\
\hline $\begin{array}{l}\text { Docente 2: Además, se supone que } \\
\text { ahí están estas dos personas pero en } \\
\text { el espa-cio que queda entre la mesa y } \\
\text { la pared también hay sillas ¿no? ¿o es } \\
\text { pared con mesa?, }\end{array}$ & $\begin{array}{l}\text { La Docente } 4 \text {, no se aproxima a la pizarra ni } \\
\text { raya la lámina, sólo se dirige al estudiante } 1 .\end{array}$ & $\begin{array}{l}\text { El estudiante } 1 \text { sólo } \\
\text { señala el espacio } \\
\text { indicado. }\end{array}$ & $\begin{array}{l}\text { La pizarra se mantiene } \\
\text { inactiva, sólo participa como } \\
\text { referencia de imagen. }\end{array}$ \\
\hline $\begin{array}{l}\text { Estudiante 4: Por eso se pensó } \\
\text { solamente en ese rincón, porque el } \\
\text { resto estaba todo ocupado (señala la } \\
\text { lámina) Ese ese era el espacio } \\
\text { disponible }\end{array}$ & $\begin{array}{l}\text { El estudiante } 1 \text { sólo escucha y asiente sobre } \\
\text { lo declarado por su compañera. }\end{array}$ & $\begin{array}{l}\text { Se centra la atención } \\
\text { sobre el comentario de } \\
\text { la alumna que no había } \\
\text { intervenido hasta ese } \\
\text { momento. }\end{array}$ & $\begin{array}{l}\text { La pizarra se mantiene } \\
\text { inactiva, sólo participa como } \\
\text { referencia }\end{array}$ \\
\hline $\begin{array}{l}\text { Docente } 2 \text { : pero... en el fondo porque } \\
\text { les comento esto, porque si dejamos el } \\
\text { croquis como está, se aprecia que hay } \\
\text { un pasillito que va directo a la ... la } \\
\text { ubicación que ustedes están determi- } \\
\text { nando (estudiante } 1 \text { raya la lámina } \\
\text { para marcar el espacio que se indica) y } \\
\text { ahí no es un pasillo porque o va a estar } \\
\text { la mesa o van a estar personas senta- } \\
\text { das ¿cierto? Entonces ya no es un es- } \\
\text { pacio que uno podría interpretar como.. } \\
\text { Ahhh mira va esto...va a una zona de } \\
\text { trabajo o va una zona de almacenaje, } \\
\text { no va a ser así, por eso es importante } \\
\text { como dice Juan Carlos, utilicen algún } \\
\text { medio para manifestar eso. }\end{array}$ & $\begin{array}{l}\text { La Docente, no se aproxima a la pizarra ni } \\
\text { raya la lámina, se dirige al estudiante } 1 \text { y al } \\
\text { estudiante } 4 \text {. El estudiante } 1 \text {, mientras la } \\
\text { docente expone, raya la lámina para reforzar } \\
\text { lo que ella dice. }\end{array}$ & $\begin{array}{l}\text { La lámina es rayada } \\
\text { para remarcar lo } \\
\text { declarado por la } \\
\text { docente, como una } \\
\text { manera de comunicarle } \\
\text { que la está entendiendo. }\end{array}$ & $\begin{array}{l}\text { Nuevamente un acto de } \\
\text { posicionamiento sobre el } \\
\text { trabajo realizado de parte } \\
\text { del estudiante } 1\end{array}$ \\
\hline
\end{tabular}




\begin{tabular}{|c|c|c|c|}
\hline Interacción del estudiante/docente & Paralenguaje & Pizarra Digital & Comentarios y relaciones \\
\hline $\begin{array}{l}\text { Docente 1: ¿Cuáles son los espacios } \\
\text { de accesibilidad?... (docente } 1 \text { pide el } \\
\text { lápiz al estudiante } 1 \text { y comienza a } \\
\text { rayar la lámina) (silencio mientras } \\
\text { escribe) No solamente el espacio y } \\
\text { cómo lo podrán ocupar, sino cómo } \\
\text { llego a ese espacio ¿cierto? ...voy a } \\
\text { llegar por acá (marca) ... por acá } \\
\text { (marca) (le entrega el lápiz al } \\
\text { estudiante 1) }\end{array}$ & $\begin{array}{l}\text { Tercera intervención del docente sobre la } \\
\text { lámina, es importante destacar que cada } \\
\text { intervención ha sido "solicitando" el lápiz con } \\
\text { un gesto, lo que provoca inmediatamente el } \\
\text { protagonismo del participante en el acto } \\
\text { comunicativo, además, cada vez el rayado es } \\
\text { más libre (esquemático) y relacionado con el } \\
\text { discurso oral. }\end{array}$ & $\begin{array}{l}\text { La pizarra digital nos } \\
\text { expone dos elementos } \\
\text { relevantes: la pantalla y } \\
\text { el lápiz, y éste último } \\
\text { toma relevancia porque } \\
\text { funciona como un } \\
\text { elemento que expone al } \\
\text { protagonismo de la } \\
\text { persona que participa, } \\
\text { "ofreciendo la palabra" } \\
\text { en el acto de } \\
\text { intervención con el } \\
\text { croquis. }\end{array}$ & $\begin{array}{l}\text { La pizarra evidencia la } \\
\text { secuencialidad de las } \\
\text { intervenciones realizadas } \\
\text { por los actores, por lo que el } \\
\text { uso del lápiz comienza a } \\
\text { verse como el elemento que } \\
\text { jerarquiza el dominio en el } \\
\text { discurso dialógico. }\end{array}$ \\
\hline $\begin{array}{l}\text { Estudiante 1: Bueno el recorrido } \\
\text { normal es... aquí (indica) se encuentra } \\
\text { la puerta de entrada, (marca) eso... } \\
\text { aquí sigue ... y aquí (silva y raya), } \\
\text { (muestra con un gesto el circuito) }\end{array}$ & $\begin{array}{l}\text { El estudiante pierde timidez frente a la lámina } \\
\text { y efectúa una intervención mayor, ocupando } \\
\text { en el acto de habla, tanto acentuaciones } \\
\text { verbales, onomatopeyas, ruidos y gestos, } \\
\text { como también, un rayado más libre sobre el } \\
\text { croquis. }\end{array}$ & $\begin{array}{l}\text { La confianza que ofrece } \\
\text { un "rayado sin } \\
\text { consecuencias" (porque } \\
\text { el original permanece en } \\
\text { registro e intacto), actúa } \\
\text { como acto "liberador" en } \\
\text { cuanto a los recursos } \\
\text { que el estudiante se } \\
\text { permite usar. }\end{array}$ & $\begin{array}{l}\text { El proceso de confianza del } \\
\text { alumno sobre la potestad } \\
\text { que se otorga para intervenir } \\
\text { su croquis, se da de manera } \\
\text { gradual, en la medida que } \\
\text { observa las intervenciones } \\
\text { de los docentes y de su } \\
\text { propia autogestión para } \\
\text { reforzar las correcciones y } \\
\text { las ideas propias y } \\
\text { colectivas. }\end{array}$ \\
\hline $\begin{array}{l}\text { Docente } 2: ~ ¿ T e \text { fijas que es distinto } \\
\text { eso, a lo que se ve? Entonces... para } \\
\text { que esa cosas ... para que no se les } \\
\text { olviden luego. }\end{array}$ & $\begin{array}{l}\text { Comentario que funciona como conclusión } \\
\text { sobre el proceso discursivo realizado. }\end{array}$ & $\begin{array}{l}\text { La PDi funciona como } \\
\text { instrumento } \\
\text { mnemotécnico útil } \mathrm{y} \\
\text { evidente }\end{array}$ & $\begin{array}{l}\text { En la lámina final y sus } \\
\text { rayados convergen los } \\
\text { comentaros más importantes } \\
\text { del proceso. }\end{array}$ \\
\hline
\end{tabular}
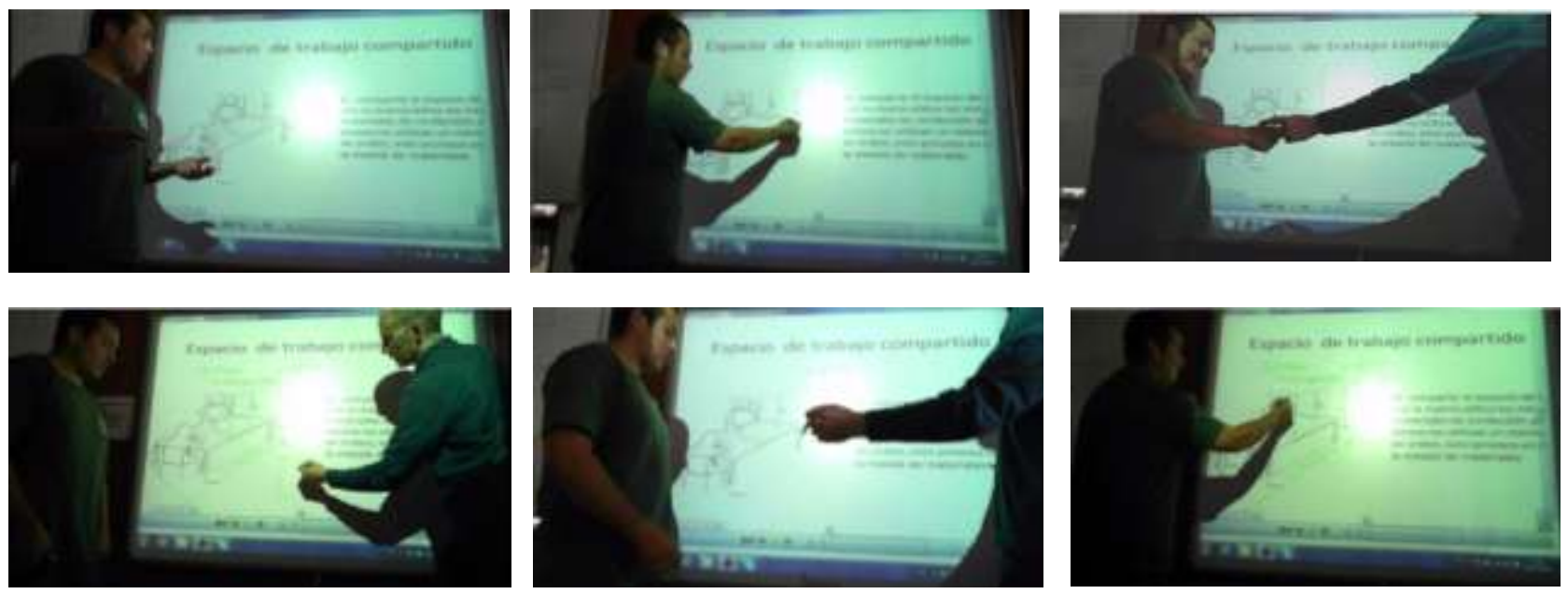

Fig. 5 Fragmento de la secuencia de uso de la Pizarra

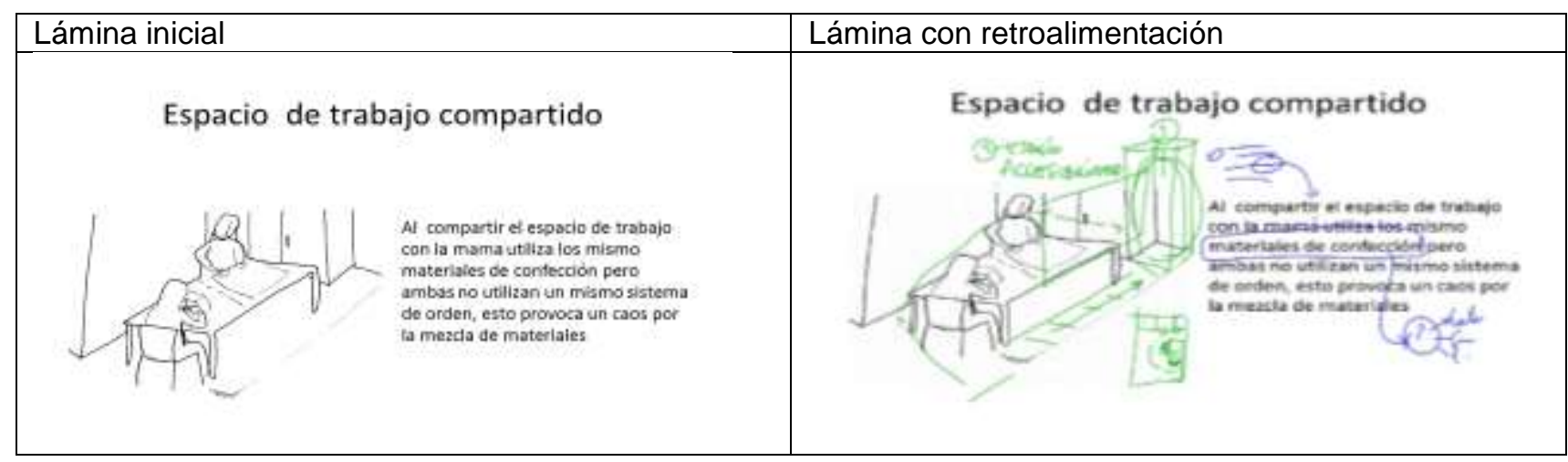

Fig. 6 Comparativa entre lámina inicial y lamina final retroalimentada 


\section{CONCLUSIONES}

El proceso de enseñanza/aprendizaje colaborativo de la observación permite un conocimiento que parte de la experiencia y que a través del diálogo, el consenso y la reflexión conjunta, reconstruye saberes en una interacción comunicativa de carácter horizontal.Cada palabra y concepto agregado se registra como una potencial fuente de valor, que ofrece nuevos alcances hacia la mejora del futuro diseño.

Como recurso tecno-pedagógico revela el ritmo de los énfasis, puesto que permite visualizar el tono, la huella de los resultados derivados de la discusión, de manera gráfica y plasmarlos en el documento individual además de compartirlos con la colectividad gracias al tamaño de visualización de la pantalla. A través del registro de los sedimentos de la conversación, de las retroalimentaciones para la toma de decisiones se favorece además, la meta-cognición y evaluación formativa del proceso observacional.

La PDI expone abiertamente al grupo las observaciones realizadas para ser comentadas y reflexionadas en conjunto, el croquis apoya y sustenta el foco de atención y es sometido a sugerencias que pueden motivar a un nuevo estudio de observación, con nuevos "filtros" que implican mayor atención a los detalles del contexto, del acto o del gesto.Permite que los participantes puedan "rayar" los bosquejos respetando el original, por lo que los autores no se sientan invadidos por un acto agresivo hacia su autoría. El "rayado" de la lámina permite generar énfasis entre lo relevante y lo superfluo según lo acordado por el conjunto de participantes en el acto de consenso, quedando registrado de manera automática, siendo al mismo tiempo una constancia de lo conversado, de los detalles significativos de los comentarios realizados por los participantes.

La PDI mantiene el curso de las correcciones como una bitacora de estudio que permite su almacenamiento y, por lo tanto, su trayecto de avance,lo que facilita el seguimiento del proceso, pudiéndose enriquecer cada corrección y redescubrir nuevos aspectos no cuestionados en el inicio.Las continuas revisiones generan la retención mnemotécnica de la observación y de sus alcances, tanto de los autores como de los participantes, quienes pueden a su vez utilizarlos para alimentar sus propias reflexiones sobre la temática observada.

Si bien el testeo preliminar de la herramienta confirma su potencial didáctico, éste se debe corroborar en estudios futuros que persigan una mayor representatividad.

\section{AGRADECIMIENTOS}

Los autores agradecen el apoyo de la Universidad del Bío-Bío a través del Proyecto de Investigación DIUBB 110404 2/R y a la comisión nacional Científica \& Tecnológica, a través de su proyecto FONDECYT código № 1121570 .

\section{REFERENCIAS}

Baelo, R., Cantón, I., Las tecnologías de la información y la comunicación en la educación superior. Estudio descriptivo y de revisión, ISSN: 1681-5653,Revista Iberoamericana de Educación, 50(7), 1-12(2009)

Beauchamp, G., Parkinson, J., Beyond the 'wow' factor: Developing interactivity with the interactive whiteboard, School Science Review, 86(316), 97-103 (2005)

Briede, Juan C. and Mora, Marcela L., Propuesta evaluativa para el Taller de Diseño Centrado en el Usuario (DCU), en la Carrera de Diseño Industrial de la Universidad del Bío-Bío, http://dx.doi.org/10.4067/S071850062013000200005.,Chile. Form. Univ., 6(2), 33-42 (2013)

Coscollola, M. D., Pizarra Digital Interactiva en el aula: Uso y valoraciones sobre el aprendizaje, ISSN: 15787001 ,Estudios Sobre Educación, 20,99-116 (2011)

Cruz, A., El Acto Arquitectónico, 1aㅡ edición, Ediciones Universitarias de Valparaíso, Pontificia Universidad Católica de Valparaíso, Valparaíso, Chile (2010)

Dorado, C., Creación de objetos de enseñanza y aprendizaje mediante el uso didáctico de la pizarra digital interactiva (PDI), ISSN: 1138-9737, Teoría de la Educación: Educación y Cultura en la Sociedad de la Información, 12(1),116-143 (2011)

Esteve, F.M. y Gisbert, M., El nuevo paradigma de aprendizaje y las nuevas tecnologías, Revista de Docencia Universitaria, ISSN:1887-4592,REDU 9(3), 55-73 (2012) 
Fernández-Cárdenas, J.M. , El habla en interacción y la calidad educativa: Los retos de la construcción de conocimiento disciplinar en ambientes mediados por tecnología digital, ISSN: 14056666, Revista Mexicana de Investigación Educativa,18(56),223-248 (2013)

Gandol, F., Carrillo, E. y Prats, M., Potencialidades y limitaciones de la Pizarra Digital Interactiva. Una revisión crítica de la literatura, ISSN: 1133-8482, Píxel-Bit. Revista de Medios y Educación, (40), 171-183 (2012)

Gallego D., Gatica N. (coords), La pizarra digital: Una ventana al mundo desde las aulas, $1^{\text {a }}$ edición, Editorial MAD, Madrid, España (2010)

Goldschmidt, G., The Dialectics of Sketching, http://dx.doi.org/10.1080/10400419109534381 ,Creativity Research Journal,4(2)123-143(1991)

Heidegger, M., La palabra, la significación de las palabras (1995) http://www.philosophia.cl/biblioteca/ Heidegger/Heidegger\%20-\%20La\%20palabra.pdf. Acceso: 15 de Febrero (2012)

Hennessy, S., The role of digital artefacts on the interactive whiteboard in supporting classroom dialogue, doi: 10.1111/j.1365-2729.2011.00416.x ,Journal of Computer Assisted Learning, 27, 463-489 (2011)

Husserl, E. , Meditaciones Cartesianas, $2^{\mathrm{a}}$ edición, Ediciones Fondo de Cultura Económica, Ciudad de México, México (1986)

Mabardi, J. F. , Maestría del Proyecto, Apuntes para la práctica de la enseñanza del proyecto, 1ํㅡㄹ edición, Ediciones Universidad del Bío-Bío,Concepción, Chile (2011)

Maznah, R. M. R., Deployment of ICT: A case for the interactive whiteboard, Malaysian Online Journal of Instructional Technology, ISSN 1449-55543, 3 (1) 78-84(2006)

Mercer, N., Hennessy S., y Paul Warwick, Using interactive whiteboards to orchestrate classroom dialogue, ISSN:1475-939X,Technology, Pedagogy and Education , 19(2)195-209(2010)

Northcote, M., Mildenhall, P., Marshall, L., Swan, P., Interactive Whiteboards: Interactive or Just Whiteboards?, Australasian Journal of Educational Technology, Vol.26 (Special issue, 4), 494-510 (2010)

Oyarzún, L., Meditaciones Estéticas, 1ํㅡㄹ Edición,Editorial Universitaria,Santiago de Chile, Chile (1981)

Prensky, M. , Digital Natives, Digital Immigrants Part 1, http://dx.doi.org/10.1108/10748120110424816 ,On the Horizon, 9 (6)1-6(2001)

Smith, K.A., Sheppard, S.D., Johnson, D.W. y Johson, R.T. ,Pedagogies of engagement: classroom-based practices, ISSN: 2168-9830, Journal of Engineering Education, 94 (1) 87-101(2005)

Thomas, J.W., A review of research on project-based learning,

http://www.k12reform.org/foundation/pbl/research (2000) Acceso : 17 diciembre (2013)

Varela, F. ,El Fenómeno de la Vida, Dolmen Ediciones, Santiago de Chile, Chile. (2000)

Warwick P., Hennessy S., Mercer N., Promoting teacher and school development through co- enquiry: developing interactive whiteboard use in a 'dialogic classroom, DOI: 10.1080/13540602.2011.554704, Teachers and Teaching, Vol. 17, Iss. 3, (2011) 